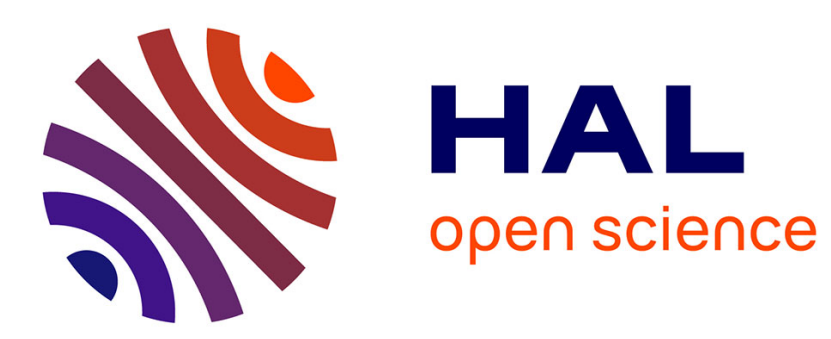

\title{
Laboratory Observations of Wave-Induced Radial Transport within an "Artificial Radiation Belt" \\ M. Mauel
}

\section{To cite this version:}

M. Mauel. Laboratory Observations of Wave-Induced Radial Transport within an "Artificial Radiation Belt". Journal de Physique IV Proceedings, 1997, 07 (C4), pp.C4-307-C4-318. 10.1051/jp4:1997425 . jpa-00255581

\section{HAL Id: jpa-00255581 https://hal.science/jpa-00255581}

Submitted on 1 Jan 1997

HAL is a multi-disciplinary open access archive for the deposit and dissemination of scientific research documents, whether they are published or not. The documents may come from teaching and research institutions in France or abroad, or from public or private research centers.
L'archive ouverte pluridisciplinaire HAL, est destinée au dépôt et à la diffusion de documents scientifiques de niveau recherche, publiés ou non, émanant des établissements d'enseignement et de recherche français ou étrangers, des laboratoires publics ou privés. 


\title{
Laboratory Observations of Wave-Induced Radial Transport within an "Artificial Radiation Belt"
}

\author{
M.E. Mauel
}

Department of Applied Physics, Columbia University, New York, NY 10027, U.S.A.

\begin{abstract}
Wave-induced radial transport of energetic electrons has been observed in a laboratory terrella. In the experiment, electron-cyclotron-resonance heating (ECRH) is used to create a localized population of trapped energetic electrons $\left(1 \mathrm{keV}<E_{h}<50 \mathrm{keV}\right)$ within a low-density discharge which we refer to as an "artificial radiation belt." As the intensity of the radiation belt increases, quasiperiodic bursts of driftresonant fluctuations, $\omega \simeq \omega_{d h}$, are excited. The frequency spectrum of this instability is time-varying and complex, and global chaotic radial transport is induced whenever the frequency spectrum is both intense and compact. High-speed measurements of the energetic electron transport are made with particle detectors, and these measurements can be directly compared with nonlinear and self-consistent simulations. We find quasilinear transport simulations do not reproduce the experimental measurements. In contrast, simulations which retain the electron's guiding-center Hamiltonian dynamics and which preserve the first, $\mu$, and second, $J$, adiabatic. invariants reproduce key temporal characteristics of the experimental measurements. The resemblance between simulation and experiment suggests that persistent phase-space structures strongly modulate the energetic electron transport and contribute to the growth and saturation of the instability.
\end{abstract}

\section{INTRODUCTION}

Since the early years of space exploration, models of fluctuation-induced radial transport of energetic particles trapped in dipolar magnetic fields have been used to describe the evolution of the planetary radiation belts [1]. When nonaxisymmetric fluctuations of geomagnetic [2] or electric [3] fields resonant with the magnetic drifts of trapped particles, the third adiabatic invariant, $\psi$, can become time-varying. Drift-resonant fluctuations mix the inner and outer regions of resonant particle distributions. In certain instances, the time-variation of $\psi$ may become stochastic [4]. Drift-resonant transport has been used to explain the profiles of the inner radiation belt [5], the injection of energetic particles during magnetic storms and substorms [6], and the radial transport coefficients of the magnetospheric ring current [7]. When combined with other, higherfrequency wave-particle interactions in the Earth's magnetosphere, the inward and outward radial transport of energetic electrons, or adiabatic "recirculation" [8], may explain the flux intensification of very energetic electrons $(E>1 \mathrm{MeV})$ at geosynchronous orbit thought to result in the failure of electronic components on spacecraft [9].

For strongly-magnetized particles within a dipole, the radial transport induced by low frequency fluctuations also provides an opportunity for detailed study of wave-particle interactions within a plasma. When the ratio of the gyroradius, $\rho$, to the equatorial radius of the particle's field line, $L$, is small, $\rho / L \ll 0.1$, the particle's three characteristic frequencies of motion, the cyclotron, $\omega_{c}$, the bounce, $\omega_{b}$, and the drift, $\omega_{d}$ frequency, separate [10]. Wave-particle interactions with low-frequency drift-resonant waves, $\omega \sim \omega_{d} \ll \omega_{b} \ll \omega_{c}$, preserve the first two adiabatic invariants [11] even when combinations of these waves create chaotic evolution of the third adiabatic invariant [12]. When collisionless energetic particles interact with low-frequency waves which preserve $\mu$ and $J$, their time-evolution can be described by a one-dimensional Vlasov fluid. Furthermore, when the particles are confined by a magnetic dipole, the relevant Hamiltonian phase-space is directly and easily observable. The canonical action is the magnetic flux, $\psi \sim 1 / L$, and the conjugate coordinate is the azimuthal angle, $\varphi$.

Article published online by EDP Sciences and available at http://dx.doi.org/10.1051/jp4:1997425 
Previously, we reported the first observations of wave-induced chaotic radial transport in a laboratory terrella, the Collisionless Terrella Experiment (CTX) [13,14]. The primary goal of CTX is to provide a laboratory test of guiding-center Hamiltonian methods used to predict and understand collisionless radial transport induced by low-frequency fluctuations in dipole-confined plasmas. By investigating the correlations between the observed transport and fluctuation spectrum and by comparing these correlations with guidingcenter simulations, we see that strong radial transport only occurs when the amplitude, frequency, and azimuthal mode number of the fluctuations meet the conditions for global chaos [14]. Greatly diminished transport is observed when the fluctuations lead to thin, radially-localized bands of chaos.

Although the presence or absence of strong radial transport can be determined by evaluating global conditions for phase-space chaos, the temporal features of the observed flux can not. We find the flux to be strongly modulated in time $(\approx 100 \%)$ near the drift frequency of the energetic electrons, $\omega_{d h}$. These modulations are related (but not equivalent) to the "drift-echoes" observed by satellites following magnetic storms and substorms $[15,16]$. In CTX, the temporal modulations of the energetic electron flux can be reproduced by computing the induced Hamiltonian phase-space flows as a function of time beginning with an initially axisymmetric electron distribution. The simulations show the modulations are due to circulating phase-space structures generated by resonant wave-particle interactions which are detected at a fixed location in the laboratory frame of reference.

In this paper, we present a more detailed interpretation of both the nonlinear growth and saturation of the low-frequency drift-resonant instabilities observed in CTX and the self-consistent dynamics of the energetic electrons. This description is both complete and relatively simple because (1) the low-frequency, electrostatic instabilities are flute-like, (2) the geometry of a dipole magnetic field is relatively simple to characterize, and (3) the dynamics of the energetic electrons are one-dimensional. We introduce a fully self-consistent, nonlinear model for the evolution of a quasineutral plasma consisting of cold ions and a mixture of hot and cold electrons confined by a dipole magnetic field. Representative numerical solutions are presented and compared with experimental observations.

We believe it highly significant that the nonlinear model reproduces the frequency sweeping observed experimentally. Examination of the Hamiltonian phase-space flows of the energetic electrons during the nonlinear saturation of the instability suggests that the rising tones seen experimentally are due to the inward propagation of "phase-space holes." The creation and dynamical evolution of phase-space structures including "holes" may be essential to the nonlinear saturation of certain instabilities [17, 18] and of driftturbulence [19]. For the experiments described in this paper, both the observations and the numerical simulations appear to be explained by a generalized nonlinear theory recently developed by Berk and coworkers $[20,21]$. In this theory, the growth and nonlinear saturation of collisionless resonant particle instabilities are dominated by three competing processes: (1) linear growth of the instability due to resonant particle effects, $\gamma_{L},(2)$ linear dissipation due to nonresonant effects, $\gamma_{d}$, and (3) nonlinear effects represented by the wave-particle trapping frequency, $\omega_{t r} \propto \sqrt{\Phi}$, where $\Phi$ is the fluctuating potential of the instability. For strongly unstable plasmas, $\gamma_{L} \gg \gamma_{d}$, instabilities grow rapidly and saturate when the linear growth rate is balanced by nonlinear trapping, $\omega_{t r} \sim \gamma_{L}$ [22]. On the other hand, for weakly unstable plasmas, $\gamma_{L} \sim \gamma_{d}$, nonlinear effects quickly overcome linear effects, and waves with both rising and falling frequencies are generated as the instability grows rapidly and nonlinearly to a large collective amplitude, $\omega_{t r} \sim \gamma_{L}$. At saturation, the wave frequencies continue to change as energy nonlinearly extracted from the resonant particles is balanced by nonresonant dissipation. Berk and co-workers suggest frequency sweeping to be a general nonlinear wave-particle effect. They note frequency sweeping has been observed experimentally during the saturated stages of several instabilities found in magnetized plasmas driven by energetic resonant particles. "Fishbone" instabilities [23] and toroidal Alfvén eigenmodes [24] excited by energetic ions in tokamaks are examples of drift-resonant magnetostatic instabilities where frequency sweeping occurs.

This paper is organized into five additional sections. Section 2 presents a brief description of the experimental device, diagnostics, and method used to produce an "artificial radiation belt" using microwave heating. Observations of the drift-resonant fluctuations using multiple electrostatic probes are summarized in Section 3. In Section 4, we introduce a self-consistent nonlinear model of the hot electron interchange instability (or HEI) [25] in the magnetic coordinates appropriate to an idealized dipole field. In Section 5, example solutions to the linearized and fully nonlinear descriptions of the HEI are presented. The nonlinear simulations illustrate frequency sweeping, and the computed phase-space flows of the energetic electrons clearly illustrate the inward propagation of "holes." At a prescribed nonresonant dissipation, these phase- 


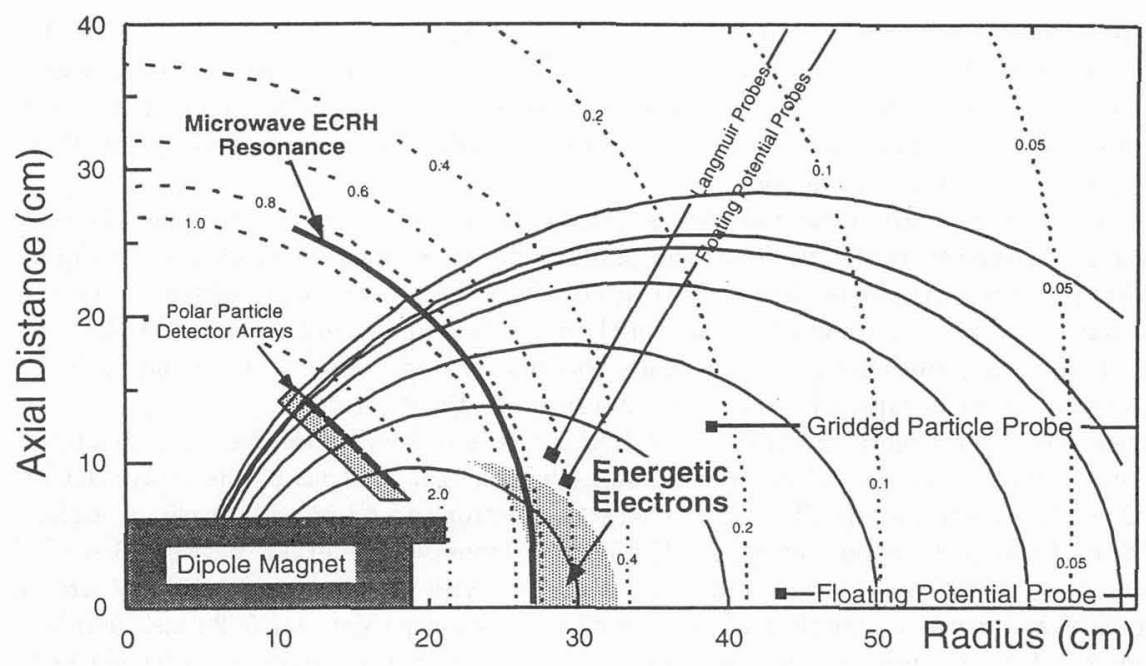

Figure 1: The magnetic field geometry of CTX. Solid lines represent magnetic field lines, and the dotted lines correspond to surfaces of constant magnetic field strength. The location of the fundamental microwave cyclotron resonance, the "artificial radiation belt", and some key diagnostics are shown.

space holes extract energetic electron energy (by causing an outward expansion of energetic electrons) while maintaining nearly constant wave amplitude. The temporal features of the energetic particle flux computed by the simulation resemble those observed experimentally. Finally, in Section 6, we summarize key conclusions and suggest new opportunities for further investigations of the nonlinear evolution of rssonant particle instabilities both in the laboratory and in space.

\section{PRODUCTION OF AN "ARTIFICIAL RADIATION BELT"}

The goal and purpose of the Collisionless Terrella Experiment (CTX) is to study the basic dynamical plasma processes which lead to collisionless radial transport of energetic particles. This is accomplished by creating an "artificial radiation belt" consisting of a population of energetic, deeply-trapped electrons as a result of cyclotron resonance absorption of microwaves within a low-density discharge.

The collisionless plasma dynamics within CTX differ from those observed in axisymmetric terrella built by Birkeland [26, 27] and Il'in' and Il'ina [28]. Birkeland's terrella were used to study untrapped chargedparticle orbits such as those followed by cosmic rays near Earth. For untrapped, very high energy particles, the normalized gyroradius satisfies the condition $\rho / L>0.3$. Il'in and Il'ina demonstrated the Dragt condition for adiabaticity (i.e. the conservation of $\mu$ ) [29], and, in their device, $\rho / L \leq 0.1$. In CTX, $\rho / L \sim 0.01$. At this normalized energy, the energetic particles in CTX are strongly trapped, adiabatic, and characterized by well-separated cyclotron, bounce, and drift frequencies.

Although the energetic electrons produced in CTX are referred to as a "radiation belt," the plasmas created within CTX do not simulate planetary magnetospheres. This is because the effects of electronplasma and electron-neutral collisions have been minimized by decreasing the plasma density and increasing the magnetic field. Although electrons are energetic, $1 \mathrm{keV}<E_{h} \leq 20 \mathrm{keV}$, the total plasma beta is low, $\beta \simeq 1 \%$. The low plasma density allows the energetic electrons to execute thousands of drift-orbits within a collision time, $\omega_{d h} \tau_{c o l} \sim 10^{4}$, but the first Alfvén field line resonance occurs at high-frequency, near $V_{A} / L \sim 50 \mathrm{MHz} \gg \omega_{d h} \sim 1 \mathrm{MHz}$. In the laboratory, low-frequency drift-resonant fluctuations are electrostatic; whereas, in the Earth's magnetosphere they are Alfvénic.

Plasma is created in CTX using a $2.45 \mathrm{GHz} 1 \mathrm{~kW}$ microwave power source illuminating a high-field water-cooled electromagnet suspended mechanically in an axisymmetric vacuum chamber approximately $140 \mathrm{~cm}$ in diameter. The magnet is surrounded by a stainless steel enclosure electrically grounded to the vacuum chamber. The magnetic field strength reaches $15 \mathrm{kG}$ at the pole faces and falls to $50 \mathrm{G}$ at the outer 
vacuum vessel wall. As shown in Figure 1, the fundamental microwave cyclotron resonance, $B_{0} \equiv B \simeq 875$ $\mathrm{G}$, intersects nearly all flux surfaces of the dipole magnet, but only electrons which mirror near $L_{0} \equiv L \simeq 30$ $\mathrm{cm}$ absorb microwave energy continuously and reach high energy. Although the CTX device is capable of long pulse discharges, the microwave discharges are usually pulsed for periods slightly less than one second which corresponds to peak x-ray emission.

The energetic electrons are measured with a krypton proportional counter viewing the equatorial midplane of the terrella and several XUV diodes and photodiodes viewing cords parallel to the dipole axis but at different radial positions. At one of the poles, arrays of biased Faraday cups, localized net current detectors, and gridded particle analyzers are used to monitor the azimuthal and radial evolution of plasma and electron profiles [30]. Langmuir probes are able to measure the density and temperature of the cooler plasma at all locations except at those occupied by the most intense energetic electrons.

The intensity of the energetic electron population is characterized by the hard $x$-ray emission produced by electron-ion and electron-neutral bremsstrahlung. Pulse-height analysis of the $x$-rays detected with the proportional counter show the distribution of energetic electrons to be non-Maxwellian, a characteristic of microwave-heated mirror-trapped electrons [31]. The electrons with energies between 1-10 keV are often referred to as the "warm" population and those electrons with energies above $10 \mathrm{keV}$ are called "hot". When the microwave power is switched off, the "hot" population persists for 5 20 ms, defining a relatively long "afterglow." By inserting probes into the region occupied by the "artificial radiation belt," the x-ray intensity decreases rapidly, and this information is used to estimate the belt's spatial extent. Additional information describing the plasma and the energetic electrons has appeared elsewhere $[13,14,30]$.

\section{OBSERVATION OF DRIFT-RESONANT FLUCTUATIONS}

When intense energetic electrons are produced, drift-resonant fluctuations $\left(\omega \sim \omega_{d h}\right)$ are observed both while the ECR heating is on and in the afterglow. During microwave heating, the fluctuations appear in quasiperiodic bursts lasting approximately $300-500 \mu \mathrm{s}$. During the afterglow, the drift-resonant oscillations persist for several milliseconds. These electrostatic fluctuations are simultaneously measured with four movable probes with high-impedance tips and matched, low-noise, wide-band preamplifiers. The observed frequencies correspond to the drift-frequencies of both the "warm" and the "hot" energetic electrons, 0.1 $\mathrm{MHz} \leq f \leq 20 \mathrm{MHz}$. The saturated wave amplitudes typically range from 100-200 V.

Correlations between multiple probes show that the waves (1) propagate azimuthally in the direction of the electron $\nabla B$ drift, (2) are flute-like with constant phase along a field line, and (3) have a broad radial structure extending throughout the plasma [32]. Fourier analysis shows the fluctuations have a timevarying and complex frequency spectrum consisting of several coherent and sometimes incoherent modes. Figure 2 shows a spectrogram of the electrostatic fluctuations during microwave heating (showing several quasiperiodic instability bursts) and during the initial period of the hot electron afterglow. Multiple waves with rising frequencies are present. Comparing the phase differences measured between probes separated azimuthally show that the azimuthal mode number of the most intense modes is $m=1$, although large amplitude instability bursts also have waves with $m=2$, and waves observed during the afterglow have been detected with $m \leq 6$. It is observed that (1) multiple modes with different frequencies often have the same azimuthal mode structure, and (2) modes present at the same time but with different $m$ often appear to be independent since the ratio of their frequencies are not harmonically related and change in time.

Coincident with the drift-resonant fluctuations is an outward expansion of the energetic electrons. This is shown in Figure 2, where the electron flux to the movable gridded particle analyzer shows a rapid increase during the instability bursts. Measurements using the photodiode and polar detector arrays, reported elsewhere [30], support this interpretation.

Analysis of the electron transport induced by the fluctuation's time-evolving frequency spectrum results in two significant conclusions. First, the presence or absence of the induced radial flux of energetic electrons depends on the spectral characteristics of the fluctuations and not simply on the amplitude [13, 14]. Intense radial transport is observed only when the frequency spectrum of the fluctuations induced global chaotic drift-motion of the resonant energetic electrons. When the frequency spectrum of the fluctuations consists of isolated coherent modes, bands of chaotic motion are predicted to occur only in thin radial bands and greatly diminished transport is observed. Secondly, the flux of electrons impacting a small movable gridded particle detector is strongly modulated. A simple quasilinear picture of phase-space diffusion averages over phase- 


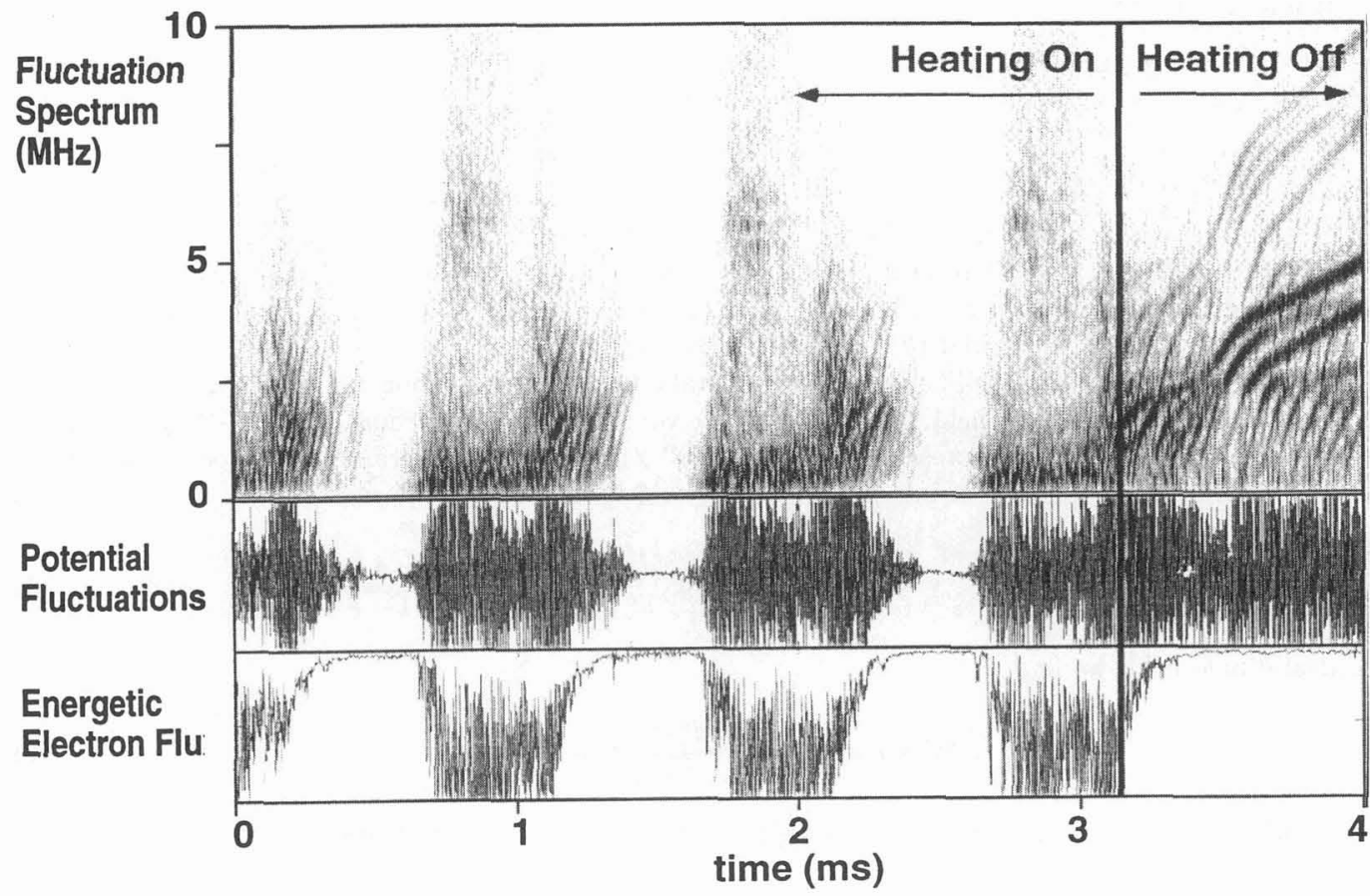

Figure 2: The measured electrostatic fluctuations and the flux of energetic electrons to the movable gridded particle detector. A spectrogram showing the time-evolving spectral amplitude of the fluctuations is also shown.

space correlations and fails to reproduce the modulated flux seen by the detector. However, a transport simulation based on the Hamiltonian motion of energetic electrons following the drift-motion induced by the measured fluctuation spectrum reproduces the frequency and modulation depth of the observations $[14,30]$.

\section{HOT ELECTRON INTERCHANGE INSTABILITY}

The analyses of collisionless transport in CTX referred to in Section 3 were based on computed particle dynamics in the presence of a collection of electrostatic waves with fixed frequencies. The wave frequencies were measured within a relatively short time interval during an instability burst when the time-variation of the spectrum could reasonably be ignored. Although the energetic electron response to these fluctuations could be understood, we did not explain why the frequency of the drift-resonant instability increases on longer time periods nor did we identify the source or describe the evolution of the phase-space structures detected by the gridded particle detector. Possible answers to these questions are addressed in this section. A fully self-consistent model for the growth and saturation of the unstable waves is introduced which includes the Hamiltonian motion of the energetic electrons to drift-resonant electrostatic fluctuations.

In the following, the basic equations used to describe flute-like electrostatic fluctuations within a dipole magnetic field are described. Since $\mu$ and $J$ are preserved, the electron dynamics are described by a twodimensional canonical phase-space, and the colder ions and electrons are described by field-line averaged particle continuity equations. When linearized, these equations describe the hot electron interchange instability (HEI) in a dipole confined plasma. Fully nonlinear solutions are computed numerically. The nonlinear solutions illustrate frequency sweeping for saturated instabilities and strong modulation of local energetic particle flux due to the transport of "phase-space holes." A more detailed description of the models presented in this section will be published elsewhere [33]. 


\subsection{Basic Equations}

In the following, we summarize a self-consistent, nonlinear model for the flute-like electrostatic hot electron interchange instability in dipole magnetic flux coordinates.

\subsubsection{The Dipole Magnetic Field}

A curl-free, axisymmetric dipole magnetic field can be represented in magnetic coordinates $\mathbf{B}=\nabla \varphi \times \nabla \psi=$ $\nabla \chi$, where $(\psi, \varphi)$ defines a field line and $\chi$ relates to a position along a field line. In spherical coordinates, $\psi=M \sin ^{2} \theta / r$, and $\chi=M \cos \theta / r^{2}$, where $M \equiv B_{0} L_{0}^{3}$ is the moment of the dipole magnet defined in terms of the field strength at an equatorial $(\theta=\pi / 2)$ reference radius, $L_{0}$.

Restricting our model to flute-like interchange instabilities, integrals along a field line incorporate the geometry of the dipole magnetic field. For example, the volume of a tube per unit flux is $\delta V(\psi)=\int d \chi / B^{2}$ with the magnetic field strength expressible as $B=\left(M / r^{3}\right) \sqrt{1+3 \cos ^{2} \theta}$. Integrals of this type are performed by first transforming the variable of integration from $\chi$ to $\xi \equiv \sin ^{2} \theta$ using the relations:

$$
r=\frac{M \xi}{\psi}, \quad \chi=\frac{\psi^{2}}{M} \sqrt{\frac{1-\xi}{\xi^{4}}}, \text { and }\left.\frac{d \chi}{d \xi}\right|_{\psi, \varphi}=\frac{\psi^{2}}{M} \frac{3 \xi-4}{2 \xi^{3} \sqrt{1-\xi}} .
$$

For a ideal dipole field. we find

$$
\delta V(\psi)=\frac{M^{3}}{\psi^{4}} \int_{0}^{1} \frac{d \xi \xi^{3}}{\sqrt{1-\xi}} \approx 0.91 \frac{M^{3}}{\psi^{4}} .
$$

The flux-tube average, $\langle A\rangle$, and the density-weighted average, $\|A\|$, are defined as

$$
\langle A\rangle \equiv \frac{1}{\delta V(\psi)} \int \frac{d \chi A}{B^{2}}, \text { and }\|A\| \equiv \frac{\langle A n\rangle}{\langle n\rangle}=\frac{1}{N} \int \frac{d \chi}{B^{2}} n A,
$$

where $n$ is the plasma density, and $N \equiv\langle n\rangle \delta V$ is the total particles on a tube per unit flux.

\subsubsection{Electron Dynamics}

We model the electrons as consisting of anisotropic energetic electrons $\left(p_{\perp} \gg p_{\|}\right)$equatorially trapped near the microwave cyclotron resonance (i.e. as shown in Figure 1) and cold electrons more uniformly distributed along the field lines. Since the instability of interest is flute-like with a frequency much higher than the cold electron drift frequency, the essential dynamics of the electrons is represented by assigning $J=0$ to the energetic electrons while preserving their total electron number density on a given flux tube. The energetic electrons form a "disk" encircling the terrella and immersed within the larger quasineutral cold plasma [14].

The interaction of nonrelativistic energetic electrons with the drift-resonant electrostatic waves are described by the guiding center drift Hamiltonian [34], $\mathcal{H}=\mu c B / e-c \Phi$, where $e$ is the magnitude of its charge, $c$ is the speed of light, $B$ is the local strength of the dipole magnetic field, $\mu \equiv m_{e} v_{\perp}^{2} / 2 B$ is the magnetic moment, $m_{e}$ is the electron mass, and $\Phi$ is the electrostatic potential. Particle motion is confined to trajectories in a two-dimensional phase-plane, $(\psi, \varphi)$, and the equations of motion take a particularly simple form:

$$
\begin{aligned}
\dot{\varphi} & =\frac{\partial \mathcal{H}}{\partial \psi}=\mu \frac{c}{e} \frac{\partial B}{\partial \psi}-c \frac{\partial \Phi}{\partial \psi} \\
\dot{\psi} & =-\frac{\partial \mathcal{H}}{\partial \varphi}=c \frac{\partial \Phi}{\partial \varphi} .
\end{aligned}
$$

For these equatorial particles, the magnetic precessional drift frequency is $\omega_{d h}=3 c \mu B / e \psi$, which scales as $\omega_{d h}=3 \mu B_{0} / m_{e} \omega_{c e 0} L^{2}$ using $\omega_{c e 0}=e B_{0} / m_{e} c$ and $L \equiv M / \psi$ to label a flux surface and a particle's equatorial radial position. Cold electrons execute radial and azimuthal $\mathbf{E} \times \mathbf{B}$ motion; whereas, the energetic electron drifts are Doppler-shifted according by the magnetic drift, $\omega_{d h}$. When $\Phi$ consists of azimuthally propagating waves, the wave-particle resonance condition, $\omega=m \omega_{d h}$, relates a particle's energy, $\mu$, to a resonant flux surface, $L \propto \sqrt{m \mu / \omega}$. As $\omega$ increases, the wave-particle drift-resonance moves inward. 
When describing the complete dynamics of a population of trapped electrons, we use the bounce-averaged distribution, $F(\mu, J, \psi, \varphi, t)$, with adiabatic constants, $(\mu, J=0)$. The collisionless evolution of $F$ is described with the following equation

$$
\frac{\partial F}{\partial t}+\frac{\partial}{\partial \varphi}(\dot{\varphi} F)+\frac{\partial}{\partial \psi}(\dot{\psi} F)=0
$$

When the particle distribution is expressed in terms of the adiabatic invariants, the number of electrons on a tube of given flux $\delta \psi \delta \varphi$ is $N_{e}=\int d \mu d J F$, and the volume-averaged electron density is $\left\langle n_{e}\right\rangle=N_{e} / \delta V(\psi)$.

\subsubsection{Electrostatic Fluctuations}

The potential fluctuations are modeled as constant along a field line and purely. electrostatic. In flux coordinates, the flux-tube-averaged form of Poisson's equation becomes

$$
\frac{\partial}{\partial \varphi}\left(h_{\varphi} \frac{\partial \Phi}{\partial \varphi}\right)+\frac{\partial}{\partial \psi}\left(h_{\psi} \frac{\partial \Phi}{\partial \psi}\right)=-4 \pi e\left(N_{i}-N_{e}\right)
$$

which determines the electrostatic potential, $\Phi(\psi, \varphi, t)$, in terms of the difference of electron and ion numbers within a flux-tube. Two geometric terms define the transformation of the Laplacian operator into field-line averaged flux-coordinates:

$$
h_{\varphi} \equiv \int \frac{d \chi}{|\nabla \psi|^{2}}=2 \frac{M}{\psi^{2}}, \text { and } h_{\psi} \equiv \int \frac{d \chi}{|\nabla \varphi|^{2}}=4 M
$$

\subsubsection{Cold Ion Dynamics}

The plasma ions are modeled as a cold, neutralizing ion-fluid convected by $\mathbf{E} \times \mathbf{B}$ and polarization drifts. This description assumes that the fluctuation frequency is much smaller than the ion cyclotron frequency, $\omega \sim \omega_{d h} / 2 \ll \omega_{c i}=e B / M_{i} c$ where $M_{i}$ is the ion mass. For drift-resonant fluctuations, this condition is equivalent to $\rho / L \ll \sqrt{m_{e} / M_{i}}$.

The field-line averaged continuity equation is

$$
\frac{\partial N_{i}}{\partial t}+\frac{\partial}{\partial \varphi}\left(N_{i}\|\nabla \varphi \cdot \mathbf{V}\|\right)+\frac{\partial}{\partial \psi}\left(N_{i}\|\nabla \psi \cdot \mathbf{V}\|\right)=0
$$

Using $\mathbf{E}=-\nabla \Phi$, the particle-conserving cold ion flow can be written as

$$
\frac{\partial N_{i}}{\partial t}+\frac{\partial}{\partial \varphi}\left[c N_{i}\left(-\frac{\partial \Phi}{\partial \psi}-\left\|\frac{|\nabla \varphi|^{2}}{\omega_{c i} B}\right\| \frac{\partial^{2} \Phi}{\partial \varphi \partial t}\right)\right]+\frac{\partial}{\partial \psi}\left[c N_{i}\left(\frac{\partial \Phi}{\partial \varphi}-\left\|\frac{|\nabla \psi|^{2}}{\omega_{c i} B}\right\| \frac{\partial^{2} \Phi}{\partial \psi \partial t}\right)\right]=0
$$

which neglects the parallel flow of plasma lost to the poles. The first term in parentheses is the $\mathbf{E} \times \mathbf{B}$ drift, and the second term is the polarization drift.

The field-line averaged ion polarization drift terms require knowledge of the density profile along a field line. This profile has not yet been measured in CTX; however, these density-weighted field-line integrals are only weakly profile dependent for a reasonable range of possible profiles. For the examples reported here, the density profile is assumed to be moderately broad along a field line, $n \sim \sqrt{\xi}=\sin \theta$, and $\left\||\nabla \varphi|^{2} / \omega_{c i} B\right\| \approx$ $0.66 M^{2} B_{0} / \psi^{4} \omega_{c i 0},\left\||\nabla \psi|^{2} / \omega_{c i} B\right\| \approx 0.77 M^{2} B_{0} / \psi^{2} \omega_{c i 0}$, where $\omega_{c i 0}$ is the ion cyclotron frequency at $B=B_{0}$.

\subsection{Linear Dispersion Relation}

The linear dispersion relation for low-frequency, flute-like modes in a hot-electron, dipole plasma can be derived from the equations presented in the previous section. We use several simplifications. First, the electron distribution is assumed to be separable with $\alpha(\psi)$ defined as the fractional density of energetic electrons. Secondly, solutions to Poisson's equation are restricted to be traveling waves localized on a flux surface where $\partial \Phi / \partial \psi \approx 0$. With these assumptions, the unperturbed electron distribution function, $F_{0}$, takes the form

$$
F_{0}(\mu, J, \psi)=N_{i 0}(\psi)[1-\alpha(\psi)] \delta(\mu) \delta(J)+N_{i 0}(\psi) \alpha(\psi) G(\mu) \delta(J)
$$


where $\int d \mu G(\mu)=1$, and the linear potential fluctuations are:

$$
\Phi(\psi, \varphi, t) \approx \Phi_{m}\left(\psi^{*}\right) \cos \left(m \varphi-\omega t+\varphi_{m}\right)\left[1-\left(\frac{\psi-\psi^{*}}{\Delta \psi}\right)^{2}+\ldots\right],
$$

with $m$ the azimuthal mode number, $\varphi_{m}$ an arbitrary phase, and $\psi^{*}=B^{*} L^{2}$ is the flux surface of peak mode amplitude (i.e. where $\partial \Phi / \partial \psi=0$ ). Only two significant terms of an expansion of the radial mode structure are retained. We refer to the radial mode width as $\Delta \psi \equiv 2 \psi^{*} / k_{L} L$, and we define the total perpendicular mode number as $m_{\perp}^{2} \equiv m^{2}+0.58\left(k_{L} L\right)^{2}$

The Fourier-Laplace transform of the linearized fluctuating electron number, $\tilde{N}_{e}$, is equal to

$$
\tilde{N}_{e}=c m \Phi_{m} \frac{1}{\omega} \frac{\partial N_{i 0}}{\partial \psi}+c m^{2} \Phi_{m} \frac{\partial\left(N_{i 0} \alpha\right)}{\partial \psi} \int d \mu \frac{\omega_{d} G}{\omega\left(\omega-m \omega_{d}\right)} .
$$

The linearized ion fluctuation number is

$$
\tilde{N}_{i}=c m \Phi_{m} \frac{1}{\omega} \frac{\partial N_{i 0}}{\partial \psi}-c m^{2} \Phi_{m}\left\|\frac{|\nabla \varphi|^{2}}{\omega_{c i} B}\right\| N_{i 0}+c \frac{\partial^{2} \Phi_{m}}{\partial \psi^{2}}\left\|\frac{|\nabla \psi|^{2}}{\omega_{c i} B}\right\| N_{i 0} .
$$

On the same surface, the field-line averaged Poisson's equation becomes

$$
\left[m^{2}+\left(k_{L} L\right)^{2}\right] \Phi_{m}=\frac{4 \pi e \psi^{* 2}}{2 M}\left(\tilde{N}_{i}-\tilde{N}_{e}\right)
$$

which defines the local dispersion relation in terms of the radial gradients of the hot and cold plasma and the distribution of energetic drift frequencies, $\omega_{d h}(\mu)$.

The hot electron interchange mode is usually described [25] in the high-density limit where $\left\langle\omega_{p i}^{2}\right\rangle / \omega_{c i}^{* 2} \approx$ $4 \pi e c N_{i 0} \psi^{*} / 0.91 M \gg 1$. For CTX, $\left\langle\omega_{p i}^{2}\right\rangle / \omega_{c i}^{* 2} \simeq 10^{3}$. When we further note that the observed instabilities have $m \sim 1$ and a broad radial structure, the local dispersion is determined by the zeros of

$$
D\left(\omega, m, m_{\perp}\right) \approx 1+1.5 \frac{m^{2}}{m_{\perp}^{2}} \frac{\psi^{*}}{N_{i 0}} \frac{\partial\left(N_{i 0} \alpha\right)}{\partial \psi} \int d \mu \frac{\omega_{c i}^{*} \omega_{d} G}{\omega\left(\omega-m \omega_{d}\right)}
$$

This dispersion relation is easily examined using two relatively simple forms for $G(\mu)$. When $G(\mu)=$ $\delta\left(\mu-\mu_{0}\right)$, the energetic electrons are mono-energetic. $D$ is a simple quadratic function of $\omega$,

$$
D \approx 1+\frac{m^{2}}{m_{\perp}^{2}} \frac{\Gamma_{h}^{2}}{\omega\left(\omega-m \omega_{d 0}\right)} .
$$

In Eq. 17, $\omega_{d 0}$ is the hot electron drift frequency, and $\Gamma_{h}^{2}=1.5 \omega_{c i}^{*} \omega_{d 0}\left(\psi^{*} / N_{i 0}\right)\left(\partial N_{i 0} \alpha / \partial \psi\right)$ is the hot electron interchange drive. This reproduces the form of the HEI dispersion relation derived by Krall [25]. Instability results whenever $\Gamma_{h}^{2}>m_{\perp}^{2} \omega_{d 0}^{2}$ corresponding to unstable coupling of the negative energy precessional mode with the positive energy drift mode. The hot electron density gradient must exceed an stability threshold due to the competition between the stabilizing azimuthal ion polarization flows and the destabilizing net radial electron flows. For unstable modes, the real frequency is $m \omega_{d 0} / 2$. Low values of $m$ and broad radial mode structures $\left(m_{\perp}^{2} \ll 1\right)$ are more unstable than higher values of $m$ or $m_{\perp}$. For mono-energetic electrons, local, linear theory does not produce a wave-particle resonance, and Eq. 17 is purely reactive.

The linear stability of energetic electrons having a distribution of energies can be investigated using a model such as

$$
G(\mu)=\frac{\mu^{l-1} l^{l}}{\mu_{0}^{l} \Gamma(l)} \exp \left(-\mu l / \mu_{0}\right)
$$

where $l$ is a positive integer. When $l=1$, Eq. 18 represents a Maxwellian distribution of energetic electrons. When $l \rightarrow \infty, G$ becomes mono-energetic. For all $l, \int d \mu \mu G=\mu_{0}$. Using Eq. 18, $\dot{D}$ can be expressed in terms of the exponential integral combined with the usual Landau prescription for evaluating the energy exchanged by the wave-particle drift-resonance, $\omega=m \omega_{d h}(\mu)$. The existence of the wave-particle resonance destabilizes the HEI (and eliminates the instability threshold) for a plasma with any unstable gradient, $d \alpha / d \psi>0$. Numerical solution of the finite-temperature dispersion relation shows the growth rate is small until the Krall instability threshold is approached; however, it is significant that distinct intervals of stability are observed in CTX even with a radially-localized population of electrons having a broad energy distribution. These stable periods implies the presence of nonresonant wave dissipation not included within Ea. 17. 

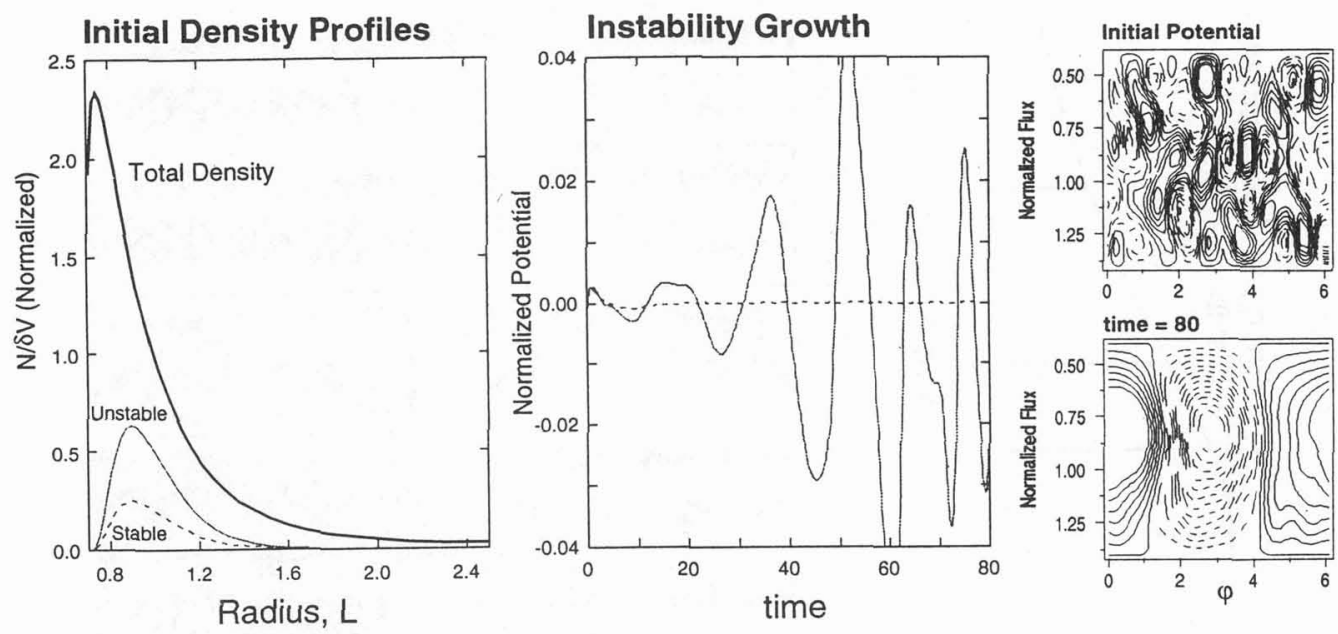

Figure 3: The evolution of the electrostatic potential during the growth and saturation of the HEI. Large amplitude waves dominated by $m=1$ grow from the initially random fluctuations when the energetic electrons are sufficiently intense. Stable and unstable hot electron densities are.shown.

\subsection{Self-Consistent Nonlinear Evolution of the HEI}

The nonlinear evolution of the hot electron interchange instability has been simulated by computing simultaneously solutions to Eqs. 5, 7 and 10. These solutions exhibit frequency chirping and strong modulation of energetic electron flux as observed in the experiment. The simulation indicates a correspondence between the rising frequency of the instability and the inward motion of phase-space "holes."

Since the self-consistent dynamics of the HEI can be described on the $(\psi, \varphi)$ phase-plane, the twodimensional time evolution of $N_{i}, \Phi$, and $F(\mu)$ can be solved using relatively standard numerical techniques on a desktop computer workstation. We incorporate an important simplification. Since $\mu$ is a constant of the motion, the electron distribution can be considered to consist of a continuum of two-dimensional phaseplanes which interact only through their mutual and collective effects on the fluctuating potential. However, the computation of the entire Vlasov time evolution of $F(\mu, t)$ would be time-consuming and unnecesșary. In the simulations reported here, we represent the hot electrons as a finite number of hot-electron phase-planes distributed at various values of $\mu, F(\mu, \varphi, \psi, t) \approx \sum_{l} g_{l}(\varphi, \psi, t) \delta\left(\mu-\mu_{l}\right)$. Since our potential fluctuations extend from the terrella to the outer vacuum chamber wall, each hot electron phase-plane resonates with the potential fluctuations at some radial location. This simplification, therefore, retains a complete description of the nonlinear wave-particle resonance without the need for excessive computation. Furthermore, the same numerical algorithm used for the evolution of the ion fluid flow can be used to compute the hot-electron phase-space flow for each value of $\mu$.

For a given electrostatic potential, $\Phi$, and its first time derivative, $\Phi^{\prime} \equiv \partial \Phi / \partial t$, the ion and electron density is advanced using a flux-corrected transport algorithm [35] modified by Zalesak [36, 37]. Zalesak demonstrated the effectiveness of this numerical technique by computing the growth of electrostatic drift instability in a plasma following a magnetospheric release of barium.

The potential fluctuations are computed by recasting Poisson's equation using an equation for the conservation of charge derived from the ion and electron dynamical equations. The ion polarization currents are used to define a field-line averaged dielectric, $\epsilon_{\varphi}(\psi), \epsilon_{\psi}(\psi)$ proportional to the axisymmetric part of the ion number density, $\bar{N}_{i} \equiv \int d \varphi N_{i} / 2 \pi$. This gives an elliptic equation for the time derivative of the potential, $\Phi^{\prime}$, in terms of the divergence of the nonaxisymmetric ion polarization currents, the $\mathbf{E} \times \mathbf{B}$ convection of the fluctuating charge density, and the magnetic precession of the hot electrons [33]. Since $\epsilon_{\varphi}$ and $\epsilon_{\psi}$ depend 

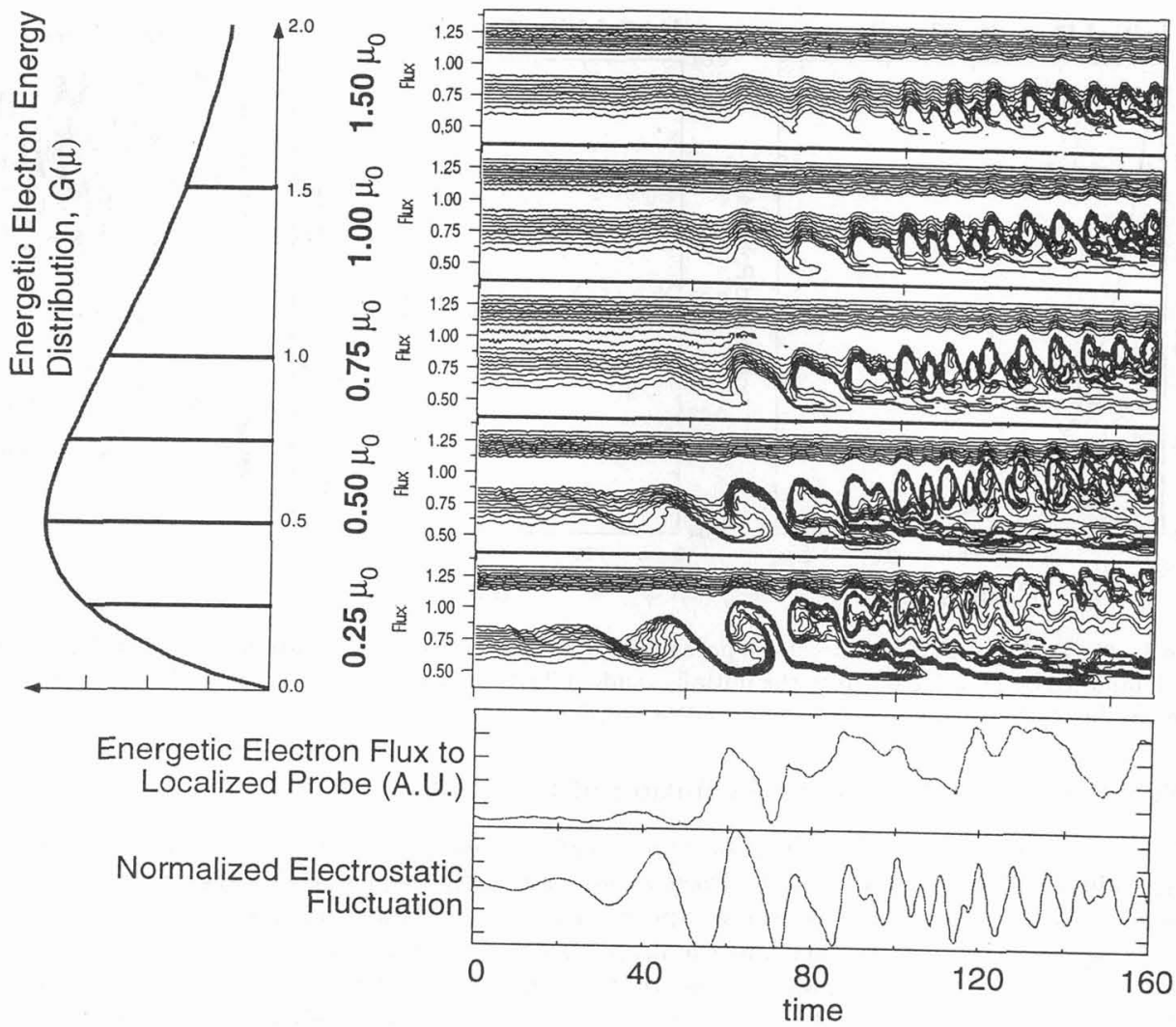

Figure 4: The evolution energetic electrons showing the modulations of the local electron flux (bottom) and the time-evolution of five hot electron "phase-planes" clearly illustrating the inward propagation of "holes" (top).

only upon $\psi$, the equation for $\Phi^{\prime}$ is solved efficiently using fast Fourier transforms of the azimuthal variations of potential and charge.

Nonresonant dissipation of the electrostatic fluctuations is required both for numerical stability and to produce frequency sweeping. Since the flux-corrected transport of electrons and ions introduces very little numerical dissipation, an explicit damping of the potential fluctuations is needed. After computing a solution for $\Phi^{\prime}$ as described above, the potential is advanced in time, from $t$ to $t+\Delta t$, according to the rule:

$$
\Phi(t+\Delta t)=\Phi(t)+\Delta t \Phi^{\prime}-(-1)^{k} \Delta t \nu \nabla^{2 k} \Phi(t)
$$

where $\nu$ is a specified constant, and $k=0,1,2, \ldots$ sets the scale-length for dissipation.

Figure 3 shows the time evolution of the electrostatic potential from a representative simulation. Initially, the potential fluctuations are small and random. If the density of energetic electrons is below a threshold set by dissipation, the fluctuations decay. As the intensity of the energetic electrons increase, rapidly growing fluctuations appear dominated by a broad, $m=1$ structure. The frequency of potential fluctuations increase as the instability saturates. The peak amplitude of the instability corresponds to a trapping frequency having $\omega_{t r} \sim \gamma_{L} \approx \omega_{d h} / 4$.

Figure 4 shows the evolution of the energetic electron phase-space distributed over five values of $\mu$ and weighted according to Eq. 18 with $l=2$. Since the initial frequency of the HEI is low, the low-energy electrons are the first to experience strong resonant diffusion. As the amplitude of the instability saturates, 
the frequency increases and electrons with higher energies begin to resonate with the waves. Since the location of the resonance changes with frequency, $\psi \propto 1 / L \propto \sqrt{\omega / m \mu}$, the drift resonance moves inward. Trapped within the resonances are low phase-space densities originating in the outer regions of the plasma. As these phase-space "holes" move inward, they displace hot and denser electrons at lower $L$ and cause transport. For the low-energy electrons, phase-space structures exist at the outer regions of the plasma which circulate at low frequency. The location of the phase-space holes within each phase-plane $(i . e$. for each value of $\mu$ ) are separated in radius but not in azimuth. As the frequency spectrum of the instability evolves, the dynamics of lower energy electrons appear chaotic. By summing the electron flux from all five phase-planes, the simulated signal from a stationary detector is strongly modulated.

\section{CONCLUSIONS AND OPPORTUNITIES}

An "artificial radiation belt" consisting of energetic electrons deeply trapped by a strong dipole electromagnet has been produced in the laboratory using microwave heating. As the intensity of the radiation belt increases, quasiperiodic bursts of hot electron interchange instability are excited which resonate with the magnetic drifts of the energetic electrons, $\omega \simeq \omega_{d h}$. High-speed measurements of the induced energetic electron transport are made with particle detectors, and these measurements show strong temporal modulations indicating the presence of circulating phase-space structures. The frequency spectrum of the nonlinear phase of the HEI is complex with slowly rising tones. We have introduced a self-consistent nonlinear simulation of the flute-like electrostatic response to a steep radial gradient of energetic electrons confined in an ideal dipole magnetic field. The simulation reproduces the temporal features of the energetic electron flux and the increasing frequency of the saturated instability. The simulation shows that a wave with rising frequency traps outer regions of low-density phase-space, called "holes," and transports these regions inward. The inner regions of high-density phase-space are displaced to lower $B$ leading to energetic electron transport. The energy released during the frequency rise does not lead to significant wave growth due to nonresonant dissipation [21].

The comparison between measurements and simulation lead to interesting questions for further investigation in the laboratory and in the Earth's magnetosphere. In the laboratory, two experiments are in progress to better measure and understand the phase-space structures created by the HEI. First, a 72-element particle analyzer is being installed at one magnetic pole in CTX in order to detect the circulation of phase-space "holes." Secondly, at the opposite pole, a high-frequency and high-power antenna has been installed in order to launch both low-frequency (electrostatic and Alfvénic) and high-frequency (whistler and electron cyclotron) waves into the plasma. These waves will provide a tool for direct interaction with the electron phase-space. For example, excitation of bounce-resonances may result in wave-induced velocity scattering and the destruction of the phase-space "holes." Finally, we can not avoid asking the questions: "Are lowfrequency waves within the Earth's magnetosphere driven unstable by energetic particles? And, if so, is frequency sweeping associated with the saturation of these instabilities?" Although most magnetospheric waves which resonate with energetic particles are externally driven by solar wind variability and plasma flows near the magnetosphere [38], theory and observation have indicated the possibility of drift-bounce resonant excitation of Pc 4-5 pulsations by energetic ring-current ions [39]. If low-frequency Alfvén waves are excited by drift-bounce resonances, they will have a relatively short perpendicular wavelength, and the detection of frequency sweeping will require multiple satellites. Unlike the HEI, localized Alfvén pulsations in space would propagate radially if their frequency were to be changed by resonant particle phase-space structures.

\section{Acknowledgment}

The author is grateful to the contributions and enlightening suggestions from H. Warren, A. Boozer, H. Berk, and B. Breizman, and of the support of AFOSR Grant F96-2097-1-0026 and NASA Grant NAWG-3539.

\section{References}

[1] M. Schulz and L. Lanzerotti, Particle Diffusion in the Rediation Belts, (Springer-Verlag, 1974).

[2] M. P. Nakada and G. D. Mead, J. Geophys. Res. 70 (1965) 4777. 
[3] T. J. Birmingham, J. Geophys. Res. 74 (1969) 2169.

[4] A. A. Chan, L. Chen, and R. B. White, Geophys. Res. Lett. 16 (1989) 1133.

[5] T. A. Farley, A. D. Thomassian, and M. Walt, Phys, Rev. Lett. 25 (1970) 47.

[6] X. Li, et al., Geophys. Res. Lett. 20 (1993) 2423.

[7] M. Chen, L. Lyons, and M. Schulz, J. Geophys. Res. 99 (1994) 5745.

[8] D. Baker, et al., Geophys. Res. Lett. 16 (1989) 559.

[9] D. Baker, et al., Geophys. Res. Lett. 21 (1994) 409.

[10] T. G. Northrup and E. Teller, Phys. Rev. 117 (1960) 215.

[11] S. Murakami, T. Sato, and A. Hasegawa, Phys. Fluids B 2 (1990) 715.

[12] H. P. Warren, A. Bhattacharjee, and M. E. Mauel, Geophys. Res. Lett. 19 (1992) 941.

[13] H. P. Warren and M. E. Mauel, Phys. Rev. Lett. 74 (1995) 1351.

[14] H. P. Warren and M. E. Mauel, Phys, Plasmas 2 (1995) 4185.

[15] L. J. Lanzerotti, C. S. Roberts, and W. L. Brown, J. Geophys. Res. 72 (1967) 5893.

[16] A. L. Vampola and A. Korth, Geophys. Res. Lett. 19 (1992) 625.

[17] H. L. Berk, C. E. Nielsen, and K. V. Roberts, Phys. Fluids 13 (1970) 980.

[18] T. O'Neil, J. H. Wfrey, and J. Malmberg, Phys. Fluids 14 (1971) 1204.

[19] P. W. Terry, P. H. Diamond, and T. S. Hahm, Phys. Fluids B 2 (1990) 2048.

[20] H. L. Berk, B. Breizman, and M. Pekker, Phys. Rev. Lett. 76 (1996) 1256.

[21] B. Breizman, et al., Phys. Plasmas 4 (1997) 1559.

[22] T. O'Niel, Phys. Fluids 8 (1965) 2255.

[23] M. F. F. Nave, et al., Nuc. Fusion 31 (1991) 697.

[24] W. W. Heidbrink and J. R. Danielson, Phys. Plasmas 1 (1994) 4120.

[25] N. A. Krall, Phys. Fluids 9 (1966) 820.

[26] D. P. Stern, Rev. of Geophys. 27 (1989) 103.

[27] C. Störmer, The Polar Aurora, (Oxford, 1995).

[28] V. D. Il'in and A. N. Il'ina, Sov. Phys. JETP 43 (1976) 661.

[29] A. J. Dragt and J. M. Finn, J. Geophys. Res. 81 (1976) 2327.

[30] H. P. Warren, et al., Phys. of Plasmas 3 (1996) 2143.

[31] M. E. Mauel, Phys. Fluids 27 (1984) 2899.

[32] M. E. Mauel and H. P. Warren, "Observation of Drift-Resonant Fluctuations in the Collisionless Terrella Experiment," in Physics of Space Plasmas (1993), eds. T. Chang and J. Jaserse, Proc. of the 1993 Cambridge Workshop in Geoplasma Physics, (MIT, 1995).

[33] Mauel, M. E., and Warren, H. P., "Hot electron interchange instability in a plasma confined by a dipole magnetic field," to be submitted to Phys. Plasmas.

[34] A. H. Boozer, Phys. Fluids 23 (1980) 904.

[35] J. P. Boris and D. L. Book, J. Comput. Phys, 20 (1976) 397.

[36] S. T. Zalesak, J. Comput. Phys, 31 (1979) 335.

[37] S. T. Zalesak, J. Comput. Phys. 40 (1981) 497.

[38] W. J. Hughes, "Magnetospheric ULF Waves: A Tutorial with a Historical Perspective," in Solar Wind Sources of Magnetospheric ULF Waves, M. J. Engebretson, K. Takahashi, M. Scholer, eds. (Geophysical Monograph, 9817, AGU, 1994).

[39] K. Takahashi, "ULF Waves in the Magnetosphere," in Rev. of Geophys, U.S: Report to the IUGG (April, 1991) 1066. 